\title{
From Evolved Stars to the Formation and Evolution of NGC 6822
}

\author{
Sima Taefi Aghdam*1, Elham Saremi ${ }^{1}$, and Atefeh Javadi ${ }^{2,1}$ \\ ${ }^{1}$ School ofAstronomy, Institute for Research in Fundamental Sciences (IPM), P. O. Box. 19395-5531, Tehran, Iran \\ ${ }^{2}$ Department of Physics, Sharif University of Technology, P. O. Box 11365-9161, Tehran, Iran
}

\begin{abstract}
NGC 6822, an isolated dwarf irregular galaxy (dIrr), due to close distance, apparent isolation, and easy observation, has been always selected as a desired candidate for studying star formation and galactic evolution, without the strong gravitational influences of other systems. To derive the SFH of NGC 6822, the method mentioned by Javadi et al. (2011) is used which is based on theoretical models coupled with color-magnitude diagrams (CMDs) by using directly the long period variable (LPV) stars which are mostly asymptotic giant branch (AGB) stars at their very late stage of evolution, as well as more massive red super-giant (RSG) stars according to their significant role to study the star formation rate (SFR). To calculate the SFH in the bar of the galaxy, we use more than $600 \mathrm{LPV}$ stars from different catalogs of variable stars that two of the most important are Whitelock et al. (2013) and Letarte et al. (2002) that they reported the main part of the LPVs and the Carbon stars, respectively, Patrick et al. (2015) announced the RSG stars. Understanding the star formation history of NGC 6822 plays an important role in comparing the galaxy history evolution with other nearby dwarf galaxies as well as studying the nature of the evolving population of galaxies which were detected in deep redshift surveys.
\end{abstract}

Keywords: stars: variables: AGB; stars: carbon; galaxies: distances and redshifts; galaxies: individual: NGC 6822; (galaxies:) Local Group: Dwarf Irregular; infrared: stars

\section{Introduction}

In the recent decade, attention to dwarf galaxies has been increased as a special tool to understand the galaxy evolution of the different morphological types of dwarf galaxies. NGC 6822, due to close distance to us and apparent isolation, has been always selected as a desired candidate for studying star formation and galactic evolution, without the strong gravitational influences of other systems (Battinelli et al., 2006). AGB stars are helpful tracers for the properties of a galaxy. They illustrate the old- and intermediate-age population (between 1 and several Gyr), whose period is mainly between 100 up to 1500 days (Goldman et al. 2019). Understanding the star formation history (SFH) of NGC 6822 plays an important role in comparing the NGC 6822 history evolution with other nearby dwarf galaxies (Saremi et al., 2019, 2020).

\section{Data}

The confirmed long period variable (LPV) stars, red super-giant (RSG) candidates, and Carbon stars (C-type), expected to be LPVs, are selected from a number of published catalogs. In 2002, Letarte et al. used the $\mathrm{CN}-\mathrm{TiO}$ technique and they found $904 \mathrm{C}$-type stars that 539 stars are in the galaxy bar. Kacharov et al. (2011) and Sibbons et al. (2015), by studying the spectral type, classified some of the AGB stars that many of them were common with C-stars confirmed by Letarte et al. (2002). Whitelock et al. (2013) selected 3 overlapping fields of a bar of NGC 6822 for monitoring in

*aylin.t.aghdam@gmail.com 
3.5 years, thus they identified 61 Mira LPVs and some long amplitude variable (LAV) stars. Battinelli \& Demers (2011) used an area in the eastern spheroid of NGC 6822 and they reported some of cyclic and semi-regular variables in this galaxy; twenty of variables are located within the optical bar of galaxy which are often common with Whitelock Group data. The RSG stars in the NGC 6822 are identified by Massey et al. (2011) and Patrick et al. (2015) with spectroscopically confirmation.

\section{Method}

In this section, we use the method mentioned by (Javadi et al., 2011) that is based on initial mass function (IMF), introduced by Kroupa (2001), coupled with color-magnitude diagram (CMD) of galaxy by using directly the LPV stars. In this method, fitting of the theoretical isochrones are used, since it allows us to obtain stellar ages and star formation rate (SFR) which they are in preferable agreement with the observational data. There are some steps to study the SFH which are the individual mass, age (look-back time, $t$ ), and pulsation duration of the LPV stars must be determined. Meanwhile, the most desirable models to satisfy our purpose are those theoretical models from the Padova group (Marigo et al., 2017). Investigating the SFR and the used method were explained thoroughly in Hamedani Golshan et al. (2017), Hashemi et al. (2017), Javadi et al. (2011, 2017, 011b), Rezaei Kh et al. (2014), Saremi et al. (2017, 2019, 2020).

\section{Discussion and On-going Work}

The SFH of NGC 6822 will be presented by using LPV stars as a tracer of the SFH of the galaxy. Figure 1, left panel, shows the CMD which is over-plotted isochrones by Marigo et al. (2017) with a distance modulus of $23.51 \pm 0.051 \mathrm{mag}$ and interstellar extinction of $A_{V}=0.77$ (Whitelock et al., 2013), and also $Z=0.0007$ based on the result of Fusco et al. (2014), who is reported in a range of $0.0001<Z<0.01$.

We have calculated the mass of detected stars for 3 metallicities by using IMF mentioned by Kroupa (2001) which is shown in figure 1, right panel. It is understood that most of the stars whose mass investigated in a range of $\log \left(M / M_{\odot}\right)=-0.1$ to 0.5 , are $\log (t)=10.2$ to 8.4yr. These stars are detected as LPV, LAV, extreme-AGB (x-AGB), C- and S-type stars.

In the following, we are going to report the SFH of the galaxy for various metallicities and discuss about the role of stellar feedback and its effect on SFR of the isolated galaxy, NGC 6822.
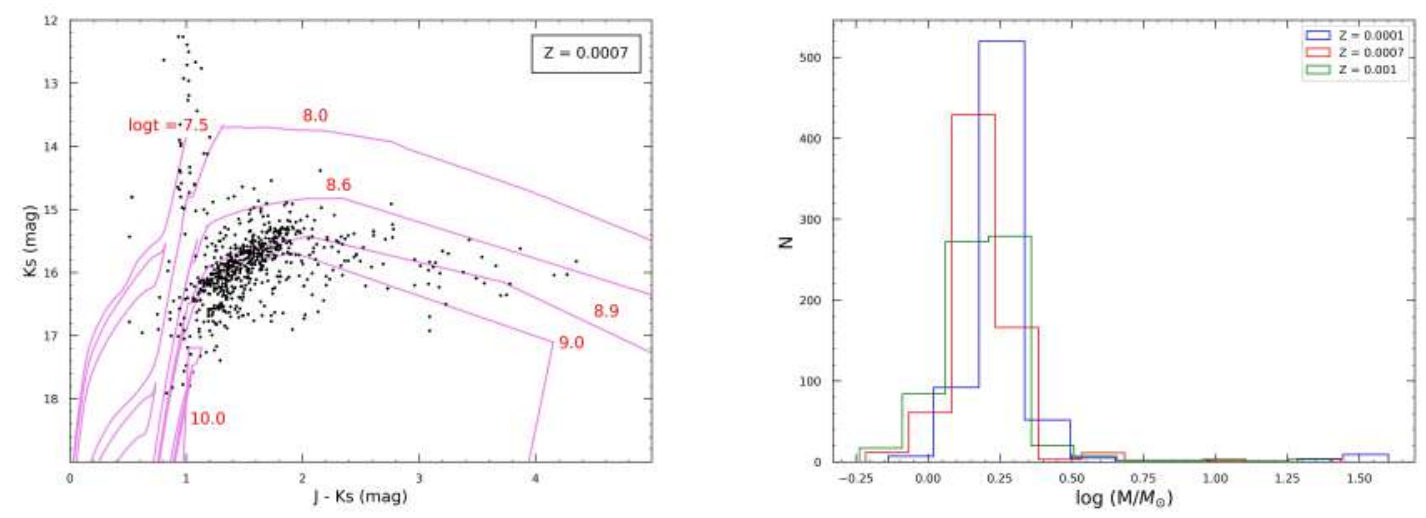

Figure 1. Left panel: overplotted $\mathrm{CMD}$ with isochrones at $\mathrm{Z}=0.0007$. Right panel: calculated mass of detected stars with theoretical isochrones in 3 different metallicities 


\section{References}

Battinelli P., Demers S., 2011, A \& A, 525, A69

Battinelli P., Demers S., Kunkel W., et al. 2006, A \& A, 451, 99

Fusco F., Buonanno R., Hidalgo S. L., et al. 2014, A \& A, 572, A26

Goldman S. R., Boyer M. L., McQuinn K. B. W., et al. 2019, ApJ, 877, 49

Hamedani Golshan R., Javadi A., van Loon J. T., et al. 2017, MNRAS, 466, 1764

Hashemi S. A., Javadi A., van Loon J. T., et al. 2017, MmSAI, 88, 436

Hashemi S. A., Javadi A., van Loon J. T., 2018, MNRAS, 483, 4751

Hashemi S. A., Javadi A., van Loon J. T., et al. 2019, IAUS, 344, 77

Javadi A., van Loon J. T., Mirtorabi M. T., 2011, MNRAS, 414, 3394

Javadi A., van Loon J. T., Khosroshahi H. G., et al. 2017, MNRAS, 464, 2103

Javadi A., van Loon J. T., Mirtorabi M. T., 2011b, ASPC, 445, 497

Kacharov N., Rejkuba M., Cioni M.-R. L., 2011, A \& A, 537, A108

Kroupa P., 2001, MNRAS, 322, 231

Letarte B., Demers S., Battinelli P., et al. 2002, Astron. J., 123, 832

Marigo P., Girardi L., Bressan A., et al. 2017, ApJ, 835, 77

Massey P., Olsen K. A. G., Hodge P. W., et al. 2011, Astron. J., 141, 28

Patrick L., Evans C. J., Davies B., et al. 2015, ApJ, 803, 14

Rezaei Kh S., Javadi A., Khosroshahi H., et al. 2014, MNRAS, 445, 2214

Saremi E., Javadi A., van Loon J. T., et al. 2017, J. Phys. Conf. Ser, 869, 012068

Saremi E., Javadi A., van Loon J. T., et al. 2019, IAUS, 344, 125

Saremi E., Javadi A., van Loon J. T., et al. 2020, ApJ, 894, 135

Sibbons L. F., Ryan S. G., Napiwotzki R., et al. 2015, A \& A, 574, A102

Whitelock P., Menzies J., Feast M., et al. 2013, MNRAS, 428, 2216 